\title{
The Effect of Size of Decompressive Craniectomy on Outcome in Deep Spontaneous Intracerebral Hemorrhage
}

\author{
Sashanka Kode ${ }^{1,2}$ Ajay Hegde ${ }^{1,3, \odot}$ Girish R. Menon ${ }^{1, \odot}$ \\ ${ }^{1}$ Department of Neurosurgery, Kasturba Medical College, Manipal \\ Academy of Higher Education, Manipal, Karnataka, India \\ ${ }^{2}$ Department of Neurosurgery, Nizams Institute of Neurosciences, \\ Hyderabad, Telangana, India \\ 3 Institute of Neurological Sciences, NHS Greater Glasgow and \\ Clyde, Glasgow, United Kingdom
}

\begin{abstract}
Address for correspondence Girish Menon, MCh, Department of Neurosurgery, Kasturba Medical College, Manipal 576104, India
\end{abstract} (e-mail: girish.menon@manipal.edu).

Indian J Neurosurg 2022;11:49-54

\begin{abstract}
Keywords

- decompressive craniectomy

- spontaneous intracerebral hemorrhage

- stroke

- mRs

- surgical outcome

Introduction Spontaneous intracerebral hemorrhage $(\mathrm{SICH})$ is one of the most devastating forms of stroke with a mortality of 30 to $40 \%$. We aimed to evaluate the effect of craniotomy size and volume of decompression on surgical outcome, complications, mortality, and morbidity in patients with supratentorial capsuloganglionic bleeds who underwent a decompressive craniectomy (DC) at our institute.

Materials and Methods It is a retrospective study done between January 2015 and December 2019. All patients with capsuloganglionic bleeds who had DC and hematoma evacuation were included in the study.

Results A total of 55 patients underwent DC for SICH at our hospital during the study period. Mean anteroposterior (AP) diameter of the bone flap was $12.42 \mathrm{~cm}$. The volume of decompression did not influence mortality and morbidity in our study but a larger AP diameter was associated with a higher incidence of hydrocephalus. A smaller craniectomy with an AP diameter of $<12 \mathrm{~cm}$ caused a lesser reduction in midline shift (MLS). Persistent postoperative MLS had a significant impact on mortality and its reduction was dependent on the size of craniectomy $(p=-0.037)$

Conclusion DC with a recommended AP diameter of 12 to $13 \mathrm{~cm}$ achieves optimal results in terms of reduction in MLS. Larger DC volume carries a higher risk of hydrocephalus and requires close follow-up.
\end{abstract}

\section{Introduction}

Spontaneous intracerebral hemorrhage $(\mathrm{SICH})$ is one of the most devastating forms of stroke with a mortality of 30 to $40 \%{ }^{1}$ Following the STICH trials ${ }^{2}$ interest in surgery for $\mathrm{SICH}$ had declined, and even to date there is no class 1 evidence on the ideal management of SICH with refractory raised intracranial pressure (ICP). However, of late there is mounting

published online November 24, 2021
DOI https://doi.org/ $10.1055 / \mathrm{s}-0041-1730102$ ISSN 2277-954X evidence in literature that minimally invasive surgery is associated with significantly improved outcomes when compared with conservative treatment and conventional surgical evacuation strategy. There has been a revived interest in decompressive craniectomy (DC) also over the past few years with several large studies and meta-analysis highlighting the superiority of DC in intracerebral hemorrhage (ICH) for reducing mortality. In fact the recent American 
Heart Association guidelines on SICH recommend that DC with or without hematoma evacuation might reduce mortality for patients with supratentorial ICH who are in a coma, have large hematomas with significant midline shift (MLS), or have elevated ICP refractory to medical management (class 2b; level of evidence C). ${ }^{2}$ The rationale for DC is based upon the Monro-Kellie doctrine and the procedure was first described by Kocher and Cushing in the early 1900s. ${ }^{3}$ DC aims to enlarge the intracranial space and expand intracranial volume with the restoration of perimesencephalic cisterns, decrease the MLS, and thereby improve cerebral compliance $^{4}$ and reduce ICP. ${ }^{5}$ The optimal size of the craniectomy, which results in maximal decompression without increasing the risk of complications, is still an enigma. The size and volume of DC have been studied in detail for ischemic stroke ${ }^{6}$ and traumatic brain injury (TBI). ${ }^{7}$ However, no attempt has been made to study the effect of craniectomy size in SICH. No level 1 evidence exists regarding the ideal size of $\mathrm{DC}^{8}$ with various studies suggesting that anteroposterior (AP) diameter of at least $8 \mathrm{~cm}^{9}$ is adequate and others suggesting that at least $15 \mathrm{~cm}$ is mandatory. ${ }^{10}$ Unlike in patients with trauma and ischemic stroke, where the primary insult results in significant cerebral edema, in SICH hematoma evacuation causes significant brain tissue collapse, which is noted intraoperatively. However, perihematomal edema, rebleed, or residual bleed may lead to delayed deterioration and DC tends to have a therapeutic role in preventing such secondary deteriorations.

Our study was based on the hypothesis that in patients with large SICH, as there tends to be a collapse of brain tissue post hematoma evacuation, a large DC might not be required, like in cases of trauma or ischemic stroke. Moreover, large DC may be harmful as intraoperative brain collapse might lead to complications like shearing of bridging veins. We aimed to evaluate the effect of craniotomy size and volume of decompression achieved on surgical outcome, complications, mortality, and morbidity in patients with supratentorial capsuloganglionic bleeds who underwent DC at our institute.

\section{Methods and Methodology}

We retrospectively reviewed records of all patients who underwent primary standard DC with hematoma evacuation for unilateral spontaneous capsuloganglionic hemorrhage at our center between January 2015 and December 2019. As per our institutional protocol, patients who had two of the following features were taken up for DC: hematoma volume greater than $60 \mathrm{~mL}, \mathrm{MLS}>10 \mathrm{~mm}$, Glasgow Coma Score (GCS) $<8$, and pupillary asymmetry. Patients with lobar hemorrhage were excluded in this study.

\section{Patient Information and Initial Management}

Patient demographic data, that is, age, sex, comorbidities, GCS at admission, were collected retrospectively from our prospectively maintained institutional ICH register. All the patients underwent nonenhanced computed tomography (CT) of the brain on admission and the images were analyzed after retrieving from our picture archiving and recovery system. On admission, all the patients were intubated given the poor GCS. Blood pressure reduction was performed in all cases with a target of $<140 \mathrm{mmHg}$ as per INTERACT II. ${ }^{11}$ Brain-specific antiedema measures were initiated in all patients. Hematoma volume was calculated from the CT brain done before surgery using the formula $A B C / 2$, where $A$ and $B$ are the perpendicular maximal diameters of the lesion and $\mathrm{C}$ is the total length in the vertical plane.

\section{Surgery and Measurement of Craniectomy}

Following the confirmation of the decision to operate-a reverse question mark-Dandy's flap was taken and the fronto-temporoparietal bone was exposed. Burr holes were placed at the key, midline, temporal floor, and behind the parietal eminence and craniotomy was performed using a high-speed cutter. The craniectomy flap was made to include subtemporal decompression, medially till 1 to $2 \mathrm{~cm}$ from the midline and posteriorly up to the posterior ear line. There was no standardization in the size of craniectomy. Complete evacuation of hematoma was attempted in all cases. Lax duroplasty was performed in all cases using a free pericranial patch. Six surgeons with various levels of experience performed the cases. The bone flap was placed in the abdomen in a subcutaneous pouch. Postoperative CT scans were performed routinely at 12 to 24 hours following surgery. CT scanning was obtained in 3-mm-slice thickness without gap or overlap. Scans were assessed for completion of hematoma removal, MLS, and size of DC.

The distance between the posterior and anterior margins of the bone defect was measured on each slice using the inner table of the skull bone. AP diameter and height of the craniectomy were measured in axial and coronal CT sections, respectively. The measurements were confirmed after three-dimensional (3D) reconstruction of the images using InVasalius version 3.1. The distance between the lower border of the craniectomy and the middle cranial fossa base was measured at the level of the uncus. The distance of the margin of the craniotomy from the midline in coronal sections was measured and the mean was used in the analysis (-Fig. 1). The MLS was assessed in the patients pre and postoperative scans at the level of foramen of Monro in axial cuts.

The volume gained by craniectomy-decompressive volume (DV)-was measured using the Eq. $1 / 2 \mathrm{ABC}$, which has a strong correlation with computed 3D volumetric assessment,

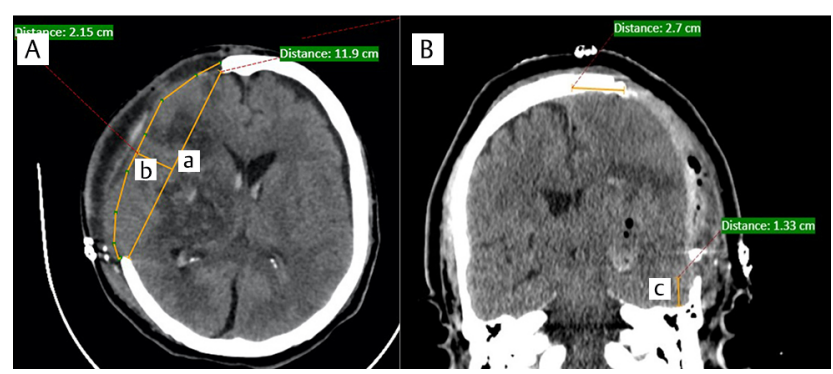

Fig. 1 Representative computed tomography scan of a brain: (A) (a) diameter of the craniectomy; (b) longest perpendicular line to the dura. Margin of the area gained by the craniectomy was hand-traced. Image B- (c) Distance from midline and base of craniectomy. 
which remains the gold standard..$^{12}$ The CT slice showing the largest diameter in AP measurement was measured as A. A perpendicular line (B) was measured from $A$ to the dural flap. The number of CT slices of the craniectomy, multiplied by the slice thickness, was the height (C) of DV (-Fig. 1). Area that was gained by DC was calculated as suggested by Nagatani et al. ${ }^{12}$

\section{Outcome}

The outcome was measured using the Modified Rankin Scale $(\mathrm{mRS})$ at discharge and 90 days. A favorable outcome was defined as mRS 0 to 3 , and a poor outcome was defined as mRS 4 to 6 .

In all patients who survived, a CT brain was performed at 6 weeks post surgery to assess hydrocephalus, subdural hygroma, and to plan replacement of bone flap. Hydrocephalus was assessed using Evan's index ${ }^{[24]}$. A value of $>0.3$ was considered significant. The following variables were analyzed for their influence on mRS, MLS, and hydrocephalus: AP length of the flap, height of the flap, and distance from the midline.

\section{Statistical Analysis}

Descriptive statistics were reported for continuous variables. Continuous variables were analyzed using independent Student's $t$-test and one-way analysis of variance for normally distributed data while Welch-Satterthwaite $t$-test was used for variables with unequal variances. Categorical variables were analyzed with the chi-squared test. Univariate Spearman's correlations were performed between all the independent variables and mRS at follow-up. Mann-Whitney $U$ test was used to analyze nonparametric data. A $5 \% \alpha$ error and $80 \% \beta$ error along with a corrected $p$-value of $<0.05$ were considered as statistically significant. The analysis was performed using IBM SPSS version 24 for Windows.

\section{Results}

A total of 63 patients underwent DC during the study period, and 55 patients were included in the study group. The mean age was $50.73 \pm 11.51$ years with a male preponderance (47:8). Mean systolic blood pressure on admission was 186.47 $\pm 29.53 \mathrm{mmHg}$ and diastolic was $104.69 \pm 17.7 \mathrm{mmHg}$. Median GCS on presentation was 8 and preoperative GCS was 7 with the volume of hematoma of $49.39 \pm 23.33 \mathrm{~mL}$. Twenty-three patients had an intraventricular extension of hematoma and 10 had hydrocephalus in the presenting scan. Mean preoperative MLS was $1.05 \pm 0.32$, mean time to surgery was 18.47 hours, and hospital stay was $15 \pm 8.12$ days ( - Table $\mathbf{1}$ ).

Mean AP diameter of the DC bone flap in our study was $12.42 \mathrm{~cm}(10.9-14.7 \mathrm{~cm})$. The AP diameter had no statistically significant impact on morbidity or mortality of the patient ( $p=0.4$ and 0.8 , respectively). The mean AP diameter of the patients who developed postoperative hydrocephalus was higher than the patients who did not $(12.8$ and $12.1 \mathrm{~cm}$, respectively; $p=0.036$ ). No similar correlation could be made with the development of postoperative hygroma $(p=0.49)$.
The mean height of the DC flap was $9.88 \pm 0.8 \mathrm{~cm}$. The patients who had a greater height of the flap had a better outcome. Mean height of DC in the patients who had a good outcome was: 10 vs. $9.2 \mathrm{~cm}(p=0.021)$. Of the 55 patients, 28 had craniotomy flush with the temporal base and 27 patients did not. In these patients the residual temporal bone from the base ranged from 4 to $20 \mathrm{~mm}$. The patients who had a craniotomy up to the temporal base had a 3-month mortality rate of $21 \%$ in comparison with $33 \%$ among the residual temporal bone group $(p=0.227)$. The mean distance from the midline to the craniotomy was $2.3 \mathrm{~cm}$ in patients who survived at 3 months compared with $2 \mathrm{~cm}$ in patients who did not. But no statistical significance was noted for the observation on overall mortality or outcome.

The volume gained by decompression was estimated by the formula $1 / 2 \mathrm{ABC}$. The mean volume was $126.67 \pm 34.78 \mathrm{~mm}^{3}$. The mean volume of craniotomy was $123.87 \pm 33.90 \mathrm{~mm}^{3}$ in patients who survived and $132.75 \pm 37.30 \mathrm{~mm}^{3}$ in patients who died $(p=0.317)$. The volume of decompression did not influence mortality or outcome ( - Tables $\mathbf{2}$ and $\mathbf{3}$ ) in our cohort.

Persisting postoperative MLS after hematoma evacuation and DC was assessed in the two groups and the patients with good outcome had an MLS of $0.34 \pm 0.24 \mathrm{~cm}$ in comparison to $0.55 \pm 0.27 \mathrm{~cm}$ in those with poor outcome $(p=0.001)$. Of the patients with persistent MLS of greater than $5 \mathrm{~mm}, 53 \%$ died in the first 3 months $(p=0.001)$. However, patients with an AP diameter of $<12 \mathrm{~cm}(14 / 55)$ had a significantly lesser reduction in MLS postoperatively ( $p$-value:-0.037).

Overall mortality in the study group at 3 months was 14 . Mean GCS and mRS on discharge were 10.34 and 4.85, respectively. Mean mRS at 90 days was 4.85 . Ten patients had a good outcome and 45 had a poor outcome at 3 months.

Table 1 Demographic and radiological data, and outcome of our cohort

\begin{tabular}{|l|l|}
\hline Parameter & $n=55$ \\
\hline Age $(y)$ & $50.73 \pm 11.51$ \\
\hline Male $(n)$ & 47 \\
\hline Female $(n)$ & 8 \\
\hline $\begin{array}{l}\text { Side }(n): \\
\text { Right }\end{array}$ & 33 \\
\hline \multicolumn{1}{|c|}{ Left } & 22 \\
\hline Volume of hematoma & $49.39 \pm 23.33 \mathrm{~mL}$ \\
\hline Preoperative midline shift & $1.05 \pm 28.262 \mathrm{~cm}$ \\
\hline Postoperative midline shift & $0.39 \pm 0.27 \mathrm{~cm}$ \\
\hline Anteroposterior diameter & $12.42 \pm 0.90 \mathrm{~cm}$ \\
\hline Height & $9.88 \pm 0.81 \mathrm{~cm}$ \\
\hline 1/2ABC (cm ${ }^{3}$ ) & $126.67 \pm 34.78$ \\
\hline Patients with craniectomy to base $(n)$ & 28 \\
\hline Complications $(n)$ & 10 \\
\hline mRS at 3 months (median) & 4 \\
\hline Mortality at 3 months $(n)$ & 14 \\
\hline
\end{tabular}

Abbreviation: mRS, Modified Rankin Scale. 
Table 2 Relationship between surgical parameters and mortality

\begin{tabular}{|l|l|l|l|}
\hline Parameter & Alive (41) & Dead (14) & $p$-Value \\
\hline AP $(\mathrm{cm})$ & $12.47 \pm 0.85$ & $12.27 \pm 1.05$ & 0.529 \\
\hline Diameter $(\mathrm{cm})$ & $1.99 \pm 0.46$ & $2.25 \pm 0.52$ & 0.116 \\
\hline Height $(\mathrm{cm})$ & $9.93 \pm 0.82$ & $9.71 \pm 0.80$ & 0.378 \\
\hline ABC/2 (mm3) & $123.87 \pm 33.90$ & $132.75 \pm 37.30$ & 0.346 \\
\hline Distance from midline (cm) & $2.14 \pm 0.49$ & $2.08 \pm 0.69$ & 0.76 \\
\hline Postoperative MLS $(\mathrm{cm})$ & $0.34 \pm 0.24$ & $0.55 \pm 0.27$ & 0.011 \\
\hline
\end{tabular}

Abbreviations: AP, anteroposterior; MLS, midline shift.

Table 3 Relationship between surgical parameters and outcome

\begin{tabular}{|l|l|l|l|}
\hline Parameter & mRS $0-3$ & mRS 4-6 & $p$-Value \\
\hline AP $(\mathrm{cm})$ & $12.47 \pm 0.85$ & $12.47 \pm 0.86$ & 0.981 \\
\hline Diameter $(\mathrm{cm})$ & $2.17 \pm 0.45$ & $1.93 \pm 0.45$ & 0.16 \\
\hline Height $(\mathrm{cm})$ & $9.35 \pm 0.70$ & $10.12 \pm 0.77$ & 0.009 \\
\hline ABC $/ 2\left(\mathrm{~mm}^{3}\right)$ & $127.10 \pm 30.21$ & $122.83 \pm 35.42$ & 0.714 \\
\hline Distance from midline $(\mathrm{cm})$ & $2.09 \pm 0.50$ & $2.16 \pm 0.49$ & 0.701 \\
\hline Postoperative MLS $(\mathrm{cm})$ & $0.23 \pm 0.22$ & $0.37 \pm 0.24$ & 0.075 \\
\hline
\end{tabular}

Abbreviations: AP, anteroposterior; MLS, midline shift; mRS, Modified Rankin Scale.

Follow-up scans were performed for all the patients at 6 weeks. Twenty-five patients had hydrocephalus (Evans index of $>0.3$ ). Also, $68 \%$ patients with an AP diameter of $>13 \mathrm{~cm}$ were observed to have hydrocephalus compared with $35.6 \%$ patients with AP diameter of $<13 \mathrm{~cm}(p=0.025$;

- Table 4). Of them, eight patients who had improvement with lumbar cerebrospinal fluid (CSF) drainage were taken up for permanent CSF diversion procedure. A subdural hygroma along the ipsilateral convexities was observed in six of these patients. The volume of craniotomy and distance from the midline did not correlate with hydrocephalus or hygroma $(p>0.05)$.

Sixteen patients had tracheostomy due to prolonged ventilatory requirement. Of the total patients, five developed postoperative meningitis, one patient had wound complication with CSF leak, and four developed ventilator-assisted pneumonia. There was no correlation between the size of craniectomy and complications.

\section{Discussion}

Surgery for ICH has been a much-debated topic in the past few decades, with multiple studies comparing conservative and surgical methods. STICH 1 and 2 trials have concluded that early surgery might have a small but clinically relevant survival advantage for patients with spontaneous superficial ICH without intraventricular hemorrhage. ${ }^{13}$ Some recent studies have opined that surgery improved survival in comparison with medical treatment with lasting benefits. ${ }^{14}$ Many centers are henceforth reverting to surgery for $\mathrm{SICH}$, but the ideal type of surgery for the best outcome has been the dilemma faced by neurosurgeons. Although minimally invasive procedures are at the forefront of surgery for SICH, DC has been found to improve mortality and morbidity outcome in several series. ${ }^{14,15} \mathrm{~A}$ recent meta-analysis of seven high-quality studies to compare DC versus craniotomy for spontaneous intracerebral bleeds has concluded that DC effectively reduced mortality in patients with SICH. ${ }^{14}$ The ideal size of the decompression required and the complications of DC in relation to the volume, dimensions, need for craniectomy up to the middle cranial fossa base, and the ideal distance from the midline have never been analyzed.

A study by Tanrikulu et al, with a cohort comprising of both TBI and SICH, concluded that a craniectomy of 12 to $18 \mathrm{~cm}$ was effective as complete hemispheric exposure (i.e., AP diameter $>18 \mathrm{~cm}$ ) in relieving intracranial hypertension. Also, a less

Table 4 Anteroposterior diameter and mortality, and postoperative outcome

\begin{tabular}{|l|l|l|l|}
\hline \multirow{2}{*}{ Parameter } & \multicolumn{1}{|c|}{ AP diameter } & \multirow{2}{*}{$p$-Value } \\
\cline { 2 - 4 } & $>13 \mathrm{~cm}$ & $<13 \mathrm{~cm}$ & 0.96 \\
\hline Mortality & $10(25.6 \%)$ & $4(25 \%)$ & 0.94 \\
\hline Outcome (mRS <3) & $17.9 \%$ & $18.8 \%$ & 0.53 \\
\hline Mean reduction in MLS & $0.52 \mathrm{~cm}$ & $0.7 \mathrm{~cm}$ & 0.025 \\
\hline Hydrocephalus & $35.9 \%$ & $68 \%$ & \\
\hline
\end{tabular}

Abbreviations: AP, anteroposterior; MLS, midline shift; mRS, Modified Rankin Scale. 
extensive approach does not increase the risk for secondary complications such as parenchymal shear stress, hemorrhage, and swelling. ${ }^{16}$ In a similar study with 526 patients undergoing DC with $21 \%$ SICH patients, the size of the bone flap did not relate either to survival or outcome ${ }^{7}$ disbanding the notion that larger the craniotomy better the outcome.

In our study, no direct correlation could be proved between AP diameter of the bone flap and outcome or mortality. The persistence of MLS, which is an indicator of raised ICP ${ }^{17}$ in the postoperative scans after DC, and incomplete hematoma evacuation were observed to have a significant impact on mortality and outcome. We observed that a diameter of 12 to $13 \mathrm{~cm}$ is adequate to achieve the required reduction in MLS. Any further increase in size is associated with complications.

Extension of DC up to the temporal base is known to improve outcome in patients with TBI and large cerebral infarcts. Few authors believe this surgical step to be more important than the size of the craniectomy flap. ${ }^{18}$ In our study, patients who had DC up to the temporal base had better survival (mortality 21 and 33\%, respectively; $p=0.157$ ), suggesting that extension up to the temporal base is a valuable addition in all cases even if the brain is lax post hematoma evacuation.

\section{Volume Gained by Decompressive Craniectomy}

The size of therapeutic DC is important in reducing refractory raised ICP. ${ }^{19}$ Several methods have been described for the analysis of the volume of decompression, with computer-assisted volumetric analysis as the gold standard. ${ }^{12}$ The mean volume gained through DC in our study was $126.64 \pm 34.78 \mathrm{~cm}^{3}$, which was concurrent with other previously done studies that had used 3D volumetric analysis. ${ }^{3}$ It has been a common belief in clinical practice that "go big or go home" is useful in DC, that is, larger the volume of decompression better the outcome. ${ }^{16}$ This was not found to be true in our study as no significant inference could be achieved to prove the benefit of a larger volume of craniectomy.

\section{Size of Craniotomy and Its Influence on Hydrocephalus}

De Bonis et al postulated that if the DC margin is too close to the midline, it reduces the external force to the bridging vein and causes an increased venous outflow to the sinus. The increased extracellular fluid absorption results in the decreased volume of the brain parenchyma and induces ventricular enlargement. Craniectomy with a superior limit too close to the midline can predispose patients to develop hydrocephalus. Hence it is recommended to perform wide DCs with the superior limit of $>25 \mathrm{~mm}$ from the midline. ${ }^{20}$ This correlation between hydrocephalus and distance from midline could not be proved in our study.

An interesting finding in our study was that patients with an AP diameter of $>13 \mathrm{~cm}$ had a significantly higher risk of developing hydrocephalus $(p=0.03)$. This was probably due to large DC playing a "flattening" role in the normally dicrotic CSF pulse wave in patients because of transmission of a pressure pulse out through the cranial defect. Arachnoid granulation function is dependent on the pressure difference between the subarachnoid space and draining venous supply. So, it is possible that disruption of pulsatile ICP dynamics secondary to opening the cranial defect results in decreased CSF outflow and absorption, thereby leading to hydrocephalus. ${ }^{21}$

\section{Limitations}

As it was a retrospective study, no randomization of the size of the craniotomy was performed preoperatively and it was left to the decision of the operating surgeon. As the cases were operated by six different surgeons with various levels of expertise, it could have played a role in the outcome.

Due to the small sample size, no specific recommendations could be made and further analysis with larger sample size is required.

\section{Conclusion}

The common belief that larger the craniectomy the better the outcome has not proven to be true in case of SICH. The recommended AP diameter of the DC is 12 to $13 \mathrm{~cm}$ to achieve best outcome with minimal complications. Larger AP diameter resulted in a higher incidence of hydrocephalus. Extension to the temporal base has better survival outcomes. Patients with persistent MLS of $>5 \mathrm{~mm}$ in the immediate postoperative scan after complete hematoma evacuation had a significantly poorer outcome and higher mortality.

\section{Funding}

None.

Note: $1 / 2 \mathrm{ABC}$ is the formula used to measure the volume gained, where $\mathrm{A}$ denotes the diameter, $\mathrm{B}$ the perpendicular line, and $C$ the height.

Note: In the expression $\mathrm{ABC} / 2, \mathrm{~A}$ and $\mathrm{B}$ are the perpendicular maximal diameters of the lesion and $C$ is the total length in the vertical plane.

Note: In the expression $\mathrm{ABC} / 2, \mathrm{~A}$ and $\mathrm{B}$ are the perpendicular maximal diameters of the lesion and $C$ is the total length in the vertical plane.

\section{Conflict of Interest}

None declared.

\section{References}

1 Dennis MS. Outcome after brain haemorrhage. Cerebrovasc Dis 2003;16(Suppl 1) :9-13

2 Hemphill JC II, Greenberg SM, Anderson CS, et al; American Heart Association Stroke Council; Council on Cardiovascular and Stroke Nursing; Council on Clinical Cardiology. Guidelines for the management of spontaneous intracerebral hemorrhage: a guideline for healthcare professionals from the American Heart Association/American Stroke Association. Stroke 2015;46(7):2032-2060

3 Olivecrona $M$, Rodling-Wahlström M, Naredi S, Koskinen L-OD. Effective ICP reduction by decompressive craniectomy in patients with severe traumatic brain injury treated by an ICP-targeted therapy. J Neurotrauma 2007;24(6):927-935

4 Bor-Seng-Shu E, Figueiredo EG, Fonoff ET, Fujimoto Y, Panerai RB, Teixeira MJ. Decompressive craniectomy and head injury: brain morphometry, ICP, cerebral hemodynamics, 
cerebral microvascular reactivity, and neurochemistry. Neurosurg Rev 2013;36(3):361-370

5 Bor-Seng-Shu E, Figueiredo EG, Amorim RLO, et al. Decompressive craniectomy: a meta-analysis of influences on intracranial pressure and cerebral perfusion pressure in the treatment of traumatic brain injury. J Neurosurg 2012;117(3):589-596

6 Jüttler E, Schwab S, Schmiedek P, et al. Decompressive Surgery for the Treatment of Malignant Infarction of the Middle Cerebral Artery (DESTINY). Stroke. Published online September 1, 2007. https://www.ahajournals.org/doi/ abs/10.1161/strokeaha.107.485649 Accessed February 05, 2021

7 Tagliaferri F, Zani G, Iaccarino C, et al. Decompressive craniectomies, facts and fiction: a retrospective analysis of 526 cases. Acta Neurochir (Wien) 2012;154(5):919-926

8 Kurzbuch AR. Does size matter? Decompressive surgery under review. Neurosurg Rev 2015;38(4):629-640

9 Kunze E, Meixensberger J, Janka M, Sörensen N, Roosen K. Decompressive craniectomy in patients with uncontrollable intracranial hypertension. Acta Neurochir Suppl (Wien) 1998;71:16-18

10 Güresir E, Schuss P, Vatter H, Raabe A, Seifert V, Beck J. Decompressive craniectomy in subarachnoid hemorrhage. Neurosurg Focus 2009;26(6):E4

11 Qureshi AI, Palesch YY, Martin R, et al. Interpretation and Implementation of Intensive Blood Pressure Reduction in Acute Cerebral Hemorrhage Trial (INTERACT II) J Vasc Interv Neurol 2014;7(2):34-40

12 Nagatani K, Wada K, Takeuchi S, et al. A suitable formula for estimating the volume gained by decompressive craniectomy in malignant hemispheric infarction. Acta Neurochir Suppl (Wien) 2013;118:285-288
13 Mendelow AD, Gregson BA, Rowan EN, Murray GD, Gholkar A, Mitchell PM; STICH II Investigators. Early surgery versus initial conservative treatment in patients with spontaneous supratentorial lobar intracerebral haematomas (STICH II): a randomised trial. Lancet 2013;382(9890):397-408

14 Yao Z, Ma L, You C, He M. Decompressive craniectomy for spontaneous intracerebral hemorrhage: a systematic review and meta-analysis. World Neurosurg 2018;110:121-128

15 Hayes SB, Benveniste RJ, Morcos JJ, Aziz-Sultan MA, Elhammady MS. Retrospective comparison of craniotomy and decompressive craniectomy for surgical evacuation of nontraumatic, supratentorial intracerebral hemorrhage. Neurosurg Focus 2013;34(5):E3

16 Tanrikulu L, Oez-Tanrikulu A, Weiss C, et al. The bigger, the better? About the size of decompressive hemicraniectomies. Clin Neurol Neurosurg 2015;135:15-21

17 Maas AI, Stocchetti N, Bullock R. Moderate and severe traumatic brain injury in adults. Lancet Neurol 2008;7(8):728-741

18 Münch E, Horn P, Schürer L, Piepgras A, Paul T, Schmiedek P. Management of severe traumatic brain injury by decompressive craniectomy. Neurosurgery 2000;47(2):315-322, discussion 322-323

19 Millar K, Eljamel S. Does size and site matter in therapeutic decompressive craniectomy? A laboratory-based experimental study. World Neurosurg 2016;95:441-446

20 De Bonis P, Pompucci A, Mangiola A, Rigante L, Anile C. Post-traumatic hydrocephalus after decompressive craniectomy: an underestimated risk factor. J Neurotrauma 2010;27(11):1965-1970

21 Waziri A, Fusco D, Mayer SA, McKhann GM II, Connolly ES Jr. Postoperative hydrocephalus in patients undergoing decompressive hemicraniectomy for ischemic or hemorrhagic stroke. Neurosurgery 2007;61(3):489-493, discussion 493-494 